Manuscript post-print for self-archiving purposes

Solar Energy Materials and Solar Cells 134, 22-28 (2015)

doi:10.1016/j.solmat.2014.11.017

\title{
Three-Dimensional Metallo-Dielectric Selective Thermal Emitters With High-Temperature Stability for Thermophotovoltaic Applications.
}

\author{
Moisés Garin ${ }^{a *}$, David Hernández ${ }^{a}$, Trifon Trifonov ${ }^{a, b}$, Ramón Alcubilla $^{a, b}$ \\ ${ }^{a}$ Grup de Recerca en Micro i Nanotecnologies, Departament d'Enginyeria Electrònica, \\ Universitat Politècnica de Catalunya, Jordi Girona 1-3 Mòdul C4, Barcelona 08034, Spain. \\ ${ }^{b}$ Centre de Recerca en Nanoenginyeria, Universitat Politècnica de Catalunya, Pascual i Vilà \\ 15, Barcelona 08028, Spain. \\ *E-mail:moises.garin@upc.edu
}

Keywords: selective thermal emitters, thermophotovoltaics, photonic crystals, macroporous silicon

\begin{abstract}
Selective thermal emitters concentrate most of their spontaneous emission in a spectral band much narrower than a blackbody. When used in a thermophovoltaic energy conversion system, they become key elements defining both its overall system efficiency and output power. Selective emitters' radiation spectra must be designed to match their accompanying photocell's band gap and, simultaneously, withstand high temperatures (above $1000 \mathrm{~K}$ ) for long operation times. The advent of nanophotonics has allowed the engineering of very selective emitters and absorbers; however, thermal stability remains a challenge since
\end{abstract}


nanostructures become unstable at temperatures much below the melting point of the used materials. In this paper we explore an hybrid 3D dielectric-metallic structure that combines the higher thermal stability of a monocrystalline 3D Silicon scaffold with the optical properties of a thin Platinum film conformally deposited on top. We show experimentally that these structures exhibit a selective emission spectrum suitable for TPV applications and that they are thermally stable at temperatures up to $1100 \mathrm{~K}$. These structures are ideal in combination with III-V semiconductors in the range $\mathrm{E}_{\mathrm{g}}=0.4-0.55 \mathrm{eV}$ such as InGaAsSb $\left(E_{\mathrm{g}}=0.5-0.6 \mathrm{eV}\right)$ and $\operatorname{InAsSbP}\left(E_{\mathrm{g}}=0.3-0.55 \mathrm{eV}\right)$.

\section{Introduction}

Thermophotovoltaic (TPV) systems [1] generate electricity from heat without any moving part. In such systems, photons thermally emitted by a high-temperature emitter are converted into electricity through a standard photovoltaic (PV) cell at near room temperature. Only those photons with energy above the PV cell's band gap $\left(E_{\mathrm{g}}\right)$ can be converted into electricity; furthermore, only those with energy slightly above $E_{\mathrm{g}}$ can be converted efficiently, as high energy photons rapidly lose their excess energy by thermalisation of the generated electron-hole pairs. As a result, high-efficiency TPV systems rely on photon filtering and recycling strategies; i.e., ways of returning useless or inefficient photons back to the emitter where they are reabsorbed helping to keep its temperature. An appealing alternative for rising efficiency is to directly avoid the radiation of inefficient photons using the so-called selective thermal emitters [2-6], which are able to radiate in a much narrower spectrum than a 
blackbody and that can be matched to the TPV cell band gap for maximum conversion efficiency.

During the last years, photonic structures with feature sizes in the order of the wavelength have demonstrated to be an excellent platform for the engineering of highly selective and customizable emitters and absorbers, owing to the unprecedented control over light they offer. For instance Yablonovitch already proposed in his pioneering work on photonic crystals [7] that spontaneous emission could be inhibited within the photonic band gap regions. Since then, several structures have been proposed for selective thermal emission in the TPV context such as 1D metal-dielectric stacks [8], 2D arrays of metallic microcavities $[4,9,10]$, and metallic 3D photonic crystals $[3,6]$. Metallic structures are typically preferred as they naturally keep the emission low at long wavelengths, while the emissivity can be enhanced at shorter wavelengths through micro-structuring.

In addition to efficiency, selective emitters must be designed to endure long operation times at high temperatures $(>1000 \mathrm{~K})$. Therefore, in order to attain the desired emission selectivity and high-temperature stability, photonic selective emitters rely on refractory metals, such as tungsten [5] and tantalum [10,11]. Unfortunately, it turns out that micro- and nano-structures are unstable at temperatures much below the melting point of these materials due to, mainly, recrystallization and surface diffusion effects $[12,13]$. Surface diffusion can rapidly wreck $2 \mathrm{D}$ or 3D microstructures [4] and lead to capillary instabilities in the case of thin films [14]. These effects can be partially alleviated by using high quality monocrystalline materials [4] and by depositing certain layers on top of the structure [6] thus reducing surface 
diffusion, although the claimed operation temperatures are still much below the melting point of the used materials.

In this paper we explore a different approach based on a 3D dielectric-metallic structure that combines the higher thermal stability of a monocrystalline 3D dielectric scaffold with the optical properties of a thin metallic film conformally deposited on top. In particular, we explore $\mathrm{Si} / \mathrm{SiO}_{2} / \mathrm{Pt}$ structures. We show experimentally that these structures exhibit a selective emission spectrum suitable for TPV applications, comparable with state-of-the-art full metallic structures, and that they are thermally stable at temperatures up to $1100 \mathrm{~K}$. These structures are well suited for TPV systems in combination with III-V semiconductors in the range $\mathrm{E}_{\mathrm{g}}=0.4-0.55 \mathrm{eV}$ such as InAsSbP $[15,16]\left(E_{\mathrm{g}}=0.3-0.55 \mathrm{eV}\right)$ and InGaAsSb $[17,18$, 19] $\left(E_{\mathrm{g}}=0.5-0.6 \mathrm{eV}\right)$.

\section{Sample fabrication}

The 3D Si scaffold microstructures were obtained by electrochemical etching of $n$-type crystalline Silicon in hydrofluoric (HF) acid solution [20]. Samples were prepared with an active area of $2.6 \mathrm{~cm}^{2}$. In contrast to colloidal crystal techniques, perfectly ordered 3D macroporous silicon structures can be fabricated in very large areas, even at full wafer scale. The fabrication process is depicted in Figure 1 . The starting material was $n$-type $\mathrm{CZ}<100>\mathrm{c}-$ Si wafers with a resistivity $\rho=0.5-0.8 \Omega \mathrm{cm}$. A $n^{+}$layer was implanted on the wafer back surface in order to work as a transparent back contact during the electrochemical etching. First of all, the pores' nucleation sites were defined through a square array (pitch of $2 \mu \mathrm{m}$ ) of 
inverted pyramids created using standard photo-lithography and anisotropic etching techniques (fig. 1a). Then, a square-array of perfectly ordered pores were electrochemically etched in 5\% HF aqueous solution, with a small amount of ethanol for wetting purposes, at a controlled solution temperature of $10^{\circ} \mathrm{C}$. During the etching, an in-depth modulation of the pores' diameter was introduced by modulating in time the etching photocurrent [21] (fig 1b). Finally, the pore walls were widened isotropically through consecutive dry-oxidation/oxideremoval steps (fig 1c,1d) until the points of maximum diameter connected laterally (fig 1e), creating the $3 \mathrm{D}$ cubic symmetry [22] (fig 1f). On each step, a $\mathrm{SiO}_{2}$ layer of $100 \mathrm{~nm}$ was grown in a tube furnace with a $70 \mathrm{~min}$. dry oxidation at $1040{ }^{\circ} \mathrm{C}$ in pure oxygen atmosphere. $\mathrm{We}$ have produced 3D cubic microstructures with a periodicity, $a$, of $2 \mu \mathrm{m}$ and a total porosity, $p$, around $70 \%$.

The fabricated 3D Si scaffolds (see Fig. 1) resemble a 3D cubic lattice of overlapped air spheroids embedded in $\mathrm{Si}$. The produced $\mathrm{Si}$ scaffold obtained in this way retains the monocrystalline nature of the original substrate, what has deep implications on its thermal stability. The monocrystalline core will remain stable up to the melting temperature of silicon due to the lack of grain boundaries; however, microstructures are still sensitive to oxygen and surface diffusion effects [23,24], which soften them in solid phase. Hopefully, both effects can be effectively suppressed under the appropriate non-oxidizing atmospheres. For instance, silicon micro- and nanostructures can withstand very high temperatures in $\mathrm{N}_{2}$ ambient for long times without any degradation, as a very thin nitride film forms over the structures which suppresses surface diffusion [25]. We appreciated no changes on the structures after $8 \mathrm{~h}$ annealing in a standard horizontal tube furnace at $1400 \mathrm{~K}$ in nitrogen ambient. We tested both, bare 3D samples and samples with $50 \mathrm{~nm}$ of grown thermal dry oxide. In fact, a thin thermal 
$\mathrm{SiO}_{2}$ layer is also known to suppress surface diffusion [25], completely freezing the microstructures at high temperatures. In addition to the excellent thermal stability, crystalline silicon has a very high thermal conductivity and is a very elastic material, what might help to relieve the thermal gradients and stresses through the 3D network without cracking.

Three-dimensional macroporous structures behave as photonic crystals [21, 22], and exhibit interesting thermal emission characteristics [26-28]; however, they are not good candidates for TPV applications since it is very difficult to efficiently reduce thermal emission at large wavelength bands [28]. In order to take advantage of the good thermal stability of crystalline silicon 3D structures we decided to deposit a conformal thin metal layer over the structure. In such metallo-dielectric structure the silicon scaffold would provide good thermal stability while the metallic film would define the optical properties, behaving much like a fullmetallic photonic structure. In particular, $5 \mathrm{~nm} \mathrm{Al}_{2} \mathrm{O}_{3}+50 \mathrm{~nm}$ Pt layers were deposited over the samples by Atomic Layer Deposition (ALD) (See Fig. 2). The thin alumina layer was not intended but required by the process, which was performed by the finish company Beneq Oy, in order to ensure a proper deposition of Platinum. The ALD technique allows for a conformal deposition, while Platinum was chosen for two main reasons namely it can be deposited by ALD and the considerably high Pt-Si eutectic temperature of $1103 \mathrm{~K}$. In order to avoid the eutectic formation and reach higher operation temperatures we investigated both depositing the $\mathrm{Pt}$ directly over $\mathrm{Si}$ and over a $30 \mathrm{~nm}$ of high-quality $\mathrm{SiO}_{2}$ layer previously grown by dry oxidation. 


\section{Thermal stability study}

The thermal stability of the fabricated structures was investigated through several annealing tests inside a home-made quartz tube furnace, able to reach temperatures up to $1200 \mathrm{~K}$, and under nitrogen ambient. Before the annealing the ambient was pumped out, down to $10^{-6} \mathrm{mbar}$, and replaced by pure nitrogen at ambient pressure. All tests lasted for eight hours at the set-point temperature, which was ramped up at $10 \mathrm{~K} / \mathrm{min}$, whereas the system was left to cool down freely at the end. Every sample ran through a single annealing cycle. The tests revealed that all samples were stable up to $1000 \mathrm{~K}$. No changes were appreciable under SEM inspection (see Fig. 3-a). After $8 \mathrm{~h}$ at $1100 \mathrm{~K}$, samples without intermediate $\mathrm{SiO}_{2}$ darkened and Pt-Si interactions could be observed under the microscope (Fig. 3-b). On the contrary, no noticeable changes were appreciable on the samples with intermediate $\mathrm{SiO}_{2}$ layer (Fig. 3-c). After $8 \mathrm{~h}$ at $1200 \mathrm{~K}$, certain regions of the samples protected with $\mathrm{SiO}_{2}$ started to show the same signs of degradation as the unprotected regions (darkening and eutectic formation), although other regions looked unaltered. We believe that this is due to the oxide barrier failure at some point, triggering the eutectic formation and progressively destroying the metallic layer. We conducted a final annealing test using a muffle furnace where we annealed the samples overnight $(12 \mathrm{~h})$ at $1273 \mathrm{~K}$ and in ambient air. We found that the Pt layers agglomerated forming droplets (Fig. 3-d) and the Si oxidized introducing mechanical stress on the samples. Interestingly, the damage in the sample without intermediate oxide layer was clearly superior, becoming mated to the naked eye and severely twisted due to induced mechanical stresses. To sum up, our tests suggest that the structures with ALD Platinum are stable up to $1100 \mathrm{~K}$ in $\mathrm{N}_{2}$ ambient when a thin high-quality $\mathrm{SiO}_{2}$ barrier is inserted between $\mathrm{Si}$ and $\mathrm{Pt}$ to avoid the eutectic formation. At higher temperatures the Pt layer will form agglomerates but will be sustained by the 3D Si scaffold. The 3D scaffold always remained, 
even though it was stretched or compressed at some regions due to the large deformations induced.

In addition to SEM inspection, we have characterized the IR specular reflectance spectra of the samples at quasi-normal $\left(13^{\circ}\right)$ incidence angle using an FT-IR spectrometer. Figure 4 shows the spectra before and after several annealing steps. The reflectance of a $50 \mathrm{~nm}$ Pt layer deposited over a polished Si plate is also included for reference purposes (curve a). As it can be seen in the figure in curves b \& c, all the samples show a flat reflectance close to one at long wavelengths, as it corresponds to a polished platinum sample. At shorter wavelengths, however, reflectance decreases abruptly and becomes close to zero, featuring several peaks and deeps. The low reflectance onset $(r=0.5)$ is at $\lambda=3 \mu \mathrm{m}$, roughly. We have observed (not shown in the figure) that the low-reflectance onset and the minimum reflectance positions slightly depends on the exact structure's porosity. Reflectance spectra of $\mathrm{Si} / \mathrm{SiO}_{2} / \mathrm{Pt}$ samples after annealing at $1100 \mathrm{~K}$ (curve c) is very close to the original ones previous to any annealing, and no difference, beyond process variations, could be observed for samples annealed at $1000 \mathrm{~K}$ or lower temperatures. Figure 4 also includes the measured reflectance of degraded samples at higher temperatures. Notice that differences between samples with and without $\mathrm{SiO}_{2}$ intermediate layer can be observed even after annealing at $1273 \mathrm{~K}$.

\section{Spectral emittance at high temperature.}

From the room-temperature quasi-normal specular reflectance the samples' emittance can be estimated by invoking the Kirchhoff's law $(e=1-r)$; which implies that our structures 
will efficiently radiate mostly at wavelengths below $3 \mu \mathrm{m}$. However, we must take into consideration that the material's optical properties might considerably differ at high temperatures $(>1000 \mathrm{~K})$ and that diffuse (and not specular) reflectance must be used when applying Kirchhoff's law [29, 30]. Consequently, we have experimentally measured the samples' normal radiance at temperatures close to $1000 \mathrm{~K}$. Figure 5 depicts schematically the thermal emission set-up. The samples were heated up to $1000 \mathrm{~K}$ in ambient air using a custom hotplate setup. The emitted thermal radiance was coupled to an FT-IR spectrometer through an intermediate image without magnification at the entrance port, where a diaphragm, set at $\varnothing=5 \mathrm{~mm}$, defined the actual sample's surface measured. The optical system fixed the solid angle of measurements at $2 \times 10^{-3} \mathrm{sr}$. Radiometric thermal emission values were attained applying a two-temperature calibration procedure and using a precision blackbody source as a reference $[31,32]$. A movable mirror allows to easily switch between sample and blackbody reference measurement. Finally, emissivity was determined by normalizing the measurements to the theoretical black-body emission at the same sample's surface temperature. The precise knowledge of the surface temperature is indeed crucial in order to obtain reliable emissivity values, and its value can sensibly deviate from the hotplate set-point. In order to minimize this calibration error, the exact sample's surface temperature was estimated through its thermal emission spectrum, relying on the spectral regions with emissivity close to one (reflectance close to zero). Additionally, a polished piece of silicon, with known gray-body emissivity, was also used to cross-check the true temperature of the sample's surface. Finally, it is worth mentioning that, although the thermal emission measurements run in ambient air, the temperatures used (up to $1000 \mathrm{~K}$ ) were not high enough to introduce any noticeable damage in the samples during their measurement, which typically lasted between one and four hours, depending on the number of exit angles measured. 
Figure 6 shows the measured radiance and emittance, in the normal direction, of a $\mathrm{Si} / \mathrm{SiO}_{2} / \mathrm{Pt}$ sample that had previously undergone an annealing test at $1100 \mathrm{~K}$, i.e. corresponding to curve $c$ in figure 3 . The estimated real surface temperature during the measurement was $939 \mathrm{~K}$. The experimental emissivity curve is in remarkably good agreement with the observed reflectance spectra $(e=1-r)$ although emittance at large wavelengths is slightly higher and tiny differences also appear at the shortest wavelengths measured, probably due to scattering of light not captured in the specular reflectance measurements. The emittance is below 0.2 for $\lambda>4 \mu \mathrm{m}(0.1$ at $\lambda=15 \mu \mathrm{m}$, beyond the scale of the figure), whereas it becomes maximum at wavelengths between $2.3 \mu \mathrm{m}$ and at $2.7 \mu \mathrm{m}$ with a maximum peak emissivity of 0.95 . For lower wavelengths, emittance decreases again down to 0.6. Notice that, since the spectral measurements are performed in ambient air, the IR absorption features of atmospheric gases introduce small artifacts on the spectra. The most notable is the doublepeak around $4.3 \mu \mathrm{m}$, which is due to the $\mathrm{CO}_{2}$ strong absorption at this frequency. Also, one of the broad absorption bands of water, in the range $5-7.5 \mu \mathrm{m}$, can be observed in this figure. In general, these artifacts can appear both as peaks or deeps of different magnitude (see Figures 4, 6 and 7) depending on the precise moment when the reference spectrum was taken.

We have also measured the sample emittance at several exit angles in order to verify that the selective emission characteristic is maintained. Angle-resolved emittance spectra was achieved by turning the sample heater $\theta$ degrees as shown in figure 5. The diaphragm aperture was slightly reduced down to $4 \mathrm{~mm}$ in order to be able to reach exit angles up to $\theta=75^{\circ}$ without the measured spot falling outside the sample's useful region. The measured spectra are shown in Figure $7 \mathrm{a}$, and the estimated temperature of the sample's surface, after 
calibration, was $889 \mathrm{~K}$. The spectra shown in the figure reveal that the emission onset is maintained with angle, although a sharp emission peak appears shifting toward longer wavelengths with increasing angle. This peak can be possibly explained by the coupling of light with a surface-like mode [33]. Nevertheless, this resonant peak do not have a significant contribution to the total radiated power. This can be better observed in the normalized radiant intensity polar plot shown in Figure $7 \mathrm{~b}$. The samples show a rather omnidirectional thermal emission profile in the high emittance band $(\lambda<3 \mu \mathrm{m})$, but with a predominant emission in the normal direction. In the high-emission edge, between 3 and $4 \mu \mathrm{m}$, a small lobe appear due to the mentioned resonant coupling. Beyond $4 \mu \mathrm{m}$, the small residual emission is very isotropic.

\section{TPV system evaluation.}

From the reported high-temperature characterization, we have evaluated the $\mathrm{Pt} / \mathrm{SiO}_{2} / \mathrm{Si}$ crystals as thermal sources for TPV applications. In a TPV system, only photons with energy above the band gap can be converted into electricity; therefore, a typical figure of merit is the emitter performance, $\eta_{E}$, defined as the ratio between the power emitted in the useful frequency band (photon energy above the gap) and the total emitted power at all frequencies. This parameter depends on the temperature of emission and the photocell bandgap. Considering our highest stable temperature $(1100 \mathrm{~K})$ and the emittance values reported at Figure 6 and 7, i.e. using the full angle-dependent emittance, our structures yield a maximum emitter efficiency $\eta_{E}=54 \%$ for a bandgap absorption edge at $\lambda_{\mathrm{g}}=3 \mu \mathrm{m}\left(E_{\mathrm{g}}=0.41 \mathrm{eV}\right)$, which is around $60 \%$ better performing than a blackbody emitter at the same temperature. Figure 8 shows the emitter efficiency of our emitter and the performance increase with respect to a BB as a function of the photocell bandgap. Interestingly, the maximum performance increase is 
reached for bandgaps between 0.4 and $0.6 \mathrm{eV}$, reaching $\eta_{E} / \eta_{B B}=1.7$ for both $E_{\mathrm{g}}=0.47$ and $E_{\mathrm{g}}=0.54 \mathrm{eV}$, even though the absolute emitter efficiency decreases monotonically for increasing $E_{\mathrm{g}}$. The proposed emitter, therefore, could be appropriate in TPV systems based on III-V compounds such as InGaAsSb $\left(E_{\mathrm{g}}=0.5-0.6 \mathrm{eV}\right)$ and $\operatorname{InAsSbP}\left(E_{\mathrm{g}}=0.3-0.55 \mathrm{eV}\right)$.

As one might notice on the light of figure 8(a), it is difficult to judge a selective emitter based only on the emitter efficiency without considering a particular TPV design context, since $\eta_{E}$ values always go higher for lower band gaps, even though this can penalize the overall system efficiency and power density. In order to get a deeper insight, we have calculated the maximum TPV system conversion efficiency achievable, in combination with our selective emitter using a detailed balance approach [34]. In order to focus on the effect of the thermal emitter, we considered no heat losses, no other mechanism for photon filtering and a 1:1 ratio between the area of the emitter and the photocells (i.e. a planar configuration). Under these constraints, we have determined the maximum theoretical efficiency for different emitter temperature operations and PV cell band gaps. Results are shown in figure 8(b) for two reference band gaps namely 0.4 and $0.5 \mathrm{eV}$. The system efficiency exhibit a very wide efficiency maximum at temperatures above $1500 \mathrm{~K}$, which is shifted toward higher temperatures for higher PV cell band gaps. For $E_{\mathrm{g}}=0.4 \mathrm{eV}$, close to the optimal, the system reaches an efficiency above $37 \%$ at $1500 \mathrm{~K}$. Notice that the highest system efficiency $(42 \%$ at around $2000 \mathrm{~K}$ ) is reached at a temperature considerably higher than the expected from simple calculations, which would be $965 \mathrm{~K}$ for blackbody peak emission at $\lambda=3 \mu \mathrm{m}$. At our highest stable temperature $(T=1100 \mathrm{~K})$, the TPV system efficiency reaches a theoretical maximum of $27.4 \%$ for $E_{\mathrm{g}}=0.4 \mathrm{eV}$ which corresponds to an output power density of $0.65 \mathrm{~W} / \mathrm{cm}^{2}$. The efficiency and power density reduces monotonically with the PV cell band 
gap, but an efficiency of $14 \%$ and an output power close to $0.35 \mathrm{~W} / \mathrm{cm}^{2}$ can still be achieved for $E_{\mathrm{g}}=0.55 \mathrm{eV}$. It is worth mentioning that the presented monocystalline silicon scaffolds can withstand temperatures close to the melting point of silicon $(1687 \mathrm{~K})$ although, at present, our structures are only stable up to $1100 \mathrm{~K}$ due to metal thin-film instabilities and failure of the $\mathrm{SiO}_{2}$ separation layer. Therefore, we believe that there is room for improvement specially investigating proper ceramic coverings in order to freeze surface diffusion and by trying different refractory metals. The $\mathrm{Si} / \mathrm{Pt}$ barrier also should be improved. The objective with our structures would be to achieve an operation temperature close to $1500 \mathrm{~K}$, where the maximum efficiency, with $E_{\mathrm{g}}=0.4 \mathrm{eV}$, would reach $38 \%$, very close to the optimum, and the output power $3.8 \mathrm{~W} / \mathrm{cm}^{2}$. In addition, at these operation temperatures the structure periodicity, and so the high emissivity edge, should be slightly tuned, allowing a slightly better performance.

\section{Conclusions}

In summary, in this work we have presented a 3D Silicon/SiO2/Pt structure, with $2 \mu \mathrm{m}$ lattice constant, easily fabricated through electrochemical etching of Silicon and ALD coating of Platinum. The structures exhibit a sharp emissivity increase at $\lambda=3 \mu \mathrm{m}$ and have been shown to be stable at temperatures up to $1100 \mathrm{~K}$ in $\mathrm{N}_{2}$ atmosphere, although the crystalline silicon 3D scaffold can withstand higher temperatures. Our structure can outperform in $70 \%$ the emitter performance of a $\mathrm{BB}$ emitter and is best performing in combination with a photocell bandgap of $0.47 \mathrm{eV}$, although reasonable efficiency improvements are expected with photocell gaps in the range $0.4-0.6 \mathrm{eV}$. This emitter can be used in combination with III-V photocells such as InGaAsSb and InAsSbP. 


\section{Acknowledgements}

This work has been partially funded by TEC2008-02520 and the Network of Excellence "Nanophotonics for Energy." Also, authors would like to thank Beneq Company for the development of Pt-coating ALD technique.

\section{References}

[1] D. L. Chubb, Fundamentals of Thermophotovoltaic Energy Conversion, Elsevier, 2007.

[2] J.-J. Greffet, R. Carminati, K. Joulain, J.-P. Mulet, S. Mainguy, Y. Chen, Coherent emission of lifght by thermal sources, Nature 416 (2002), 61-64.

[3] S. Y. Lin, J. Moreno, J. G. Fleming, Three-dimensional photonic-crystal emitter for thermal photovoltaic power generation, Appl. Phys. Lett. 83 (2003), 380-382.

[4] H. Sai, Y. Kanamori, H. Yugami, High-temperature resistive surface grating for spectral control of thermal radiation, Appl. Phys. Lett. 82 (2003), 1685-1687.

[5] Y. X. Yeng, M. Ghebrebrhan, P. Bermel, W. R. Chan, J. D. Joannopoulos, M. Soljačić, I. Celanovic, Enabling high-temperature nanophotonics for energy applications, PNAS 2012, doi: 10.1073/pnas.1120149109.

[6] K. A. Arpin, M. D. Losego, A. N. Cloud, H. Ning, J. Mallek, N. P. Sergeant, L. Zhu, Z. Yu, B. Kalanyan, G. N. Parsons, G. S. Girolami, J. R. Abelson, S. Fan, P. V. Braun, Three-dimensional selfassembled photonic crystals with high temperature stability for thermal emission modification, Nat. Commun. 4 (2013), 2630.

[7] E. Yablonovitch, Inhibited Spontaneous Emission in Solid-State Physics and Electronics, Phys. Rev. Lett. 58 (1987), 2059-2062.

[8] A. Narayanaswamy, G. Chen, Thermal emission control with one-dimensional metallodielectric photonic crystal, Phys. Rev. B 70 (2004), 125101. 
[9] I. Celanovic, N. Jovanovic, J. Kassakian, Two-dimensional tungsten photonic crystals as selective thermal emitters, Appl. Phys. Lett. 92 (2008), 193101.

[10] V. Rinnerbauer, S. Ndao, Y. X. Yeng, J. J. Senkevich, K. F. Jensen, J. D. Joannopoulos, M.

Soljačić, I. Celanovic, Large-area fabrication of high aspect ratio tantalum photonic crystals for hightemperature selective emitters, J. Vac. Sci. Technol. B 31 (2013), 011802.

[11] V. Rinnerbauer, A. Lenert, D. M. Bierman, Y. X. Yeng, W. R. Chan, R. D. Geil, J. J. Senkevich, J. D. Joannopoulos, E. N. Wang, M. Soljačić, I. Celanovic, Metallic Photonic Crystal Absorber-Emitter for Efficient Spectral Control in High-Temperature Solar Thermophotovoltaics, Adv. Energy Mater. 4 (2014), doi:10.1002/aenm.201400334.

[12] W. W. Mullins, Theory of Thermal Grooving, J. Appl. Phys. 28 (1957), 333-339.

[13] W. W. Mullins, Flattening a Nearly Plane Solid Surface due to Capillarity, J. Appl. Phys. 30 (1959), 77-83.

[14] C. V. Thompson, Solid-State Dewetting of Thin Films, Annu. Rev. Mater. Res. 42 (2012), 399_434.

[15] V. M. Andreev, V. P. Khvostikov, O. V. Khvostikova, E. V. Oliva, V. D. Rumyantsev, M. Z. Shvarts, Low-Bandgap Ge and InAsSbP/InAs-Based TPV Cells, AIP Conf. Proc. 653 (2002), 383391.

[16] C. A. Wang, Antimony-Based III-V Thermophotovoltaic Materials and Devices, AIP Conf. Proc. 738 (2004), 255-266.

[17] M. W. Dashiell et. al., Quaternary InGaAsSb Thermophotovoltaic Diodes, IEEE Trans. Elenctron. 53 (2006), 2879-2891.

[18] W. R. Chan, P. Bermel, R. C. N. Pilawa-Podgurski, C. H. Marton, K. F. Jensen, J. J. Senkevich, J. D. Joannopoulos, M. Soljačić, I. Celanovic, Toward high-energy-density, high-efficiency, and moderate-temperature chip-scale thermophotovoltaics, PNAS 110 (2013), 5309-5314. [19] A. Lenert, D. M. Bierman, Y. Nam, W. R. Chan, I. Celanovic, M. Soljačić, E. N. Wang, A nanophotonic solar thermophotovoltaic device, Nature Nanotechnology 9 (2014), 126-130. [20] V. Lehmann, The Physics of Macropore Formation in Low Doped n-Type Silicon, J. Electrochem. 
Soc. 140 (1993), 2836-2843.

[21] J. Schilling, F. Müller, S. Matthias, R. B. Wehrspohn, U. Gösele, Three-dimensional photonic crystals based on macroporous silicon with modulated pore diameter, Appl. Phys. Lett. 78 (2001), $1180-1182$.

[22] S. Matthias, F. Müller, C. Jamois, R. B. Wehrspohn, U. Gösele, Large-Area Three-Dimensional Structuring by Electrochemical Etching and Lithography, Adv. Mater. 16 (2004), 2166-2170.

[23] T. Sato, K. Mitsutake, I. Mizushima, Y. Tsunashima, Micro-structure Transformation of Silicon: A Newly Developed Transformation Technology for Patterning Silicon Surfaces using the Surface Migration of Silicon Atoms by Hydrogen Annealing, Jpn. J. Appl. Phys. 39 (2000), 5033—5038. [24] I. Mizushima, T. Sato, S. Taniguchi, Y. Tsunashima, Empty-space-in-silicon technique for fabricating a silicon-on-nothing structure, Appl. Phys. Lett. 77 (2000), 3290-3292.

[25] V. Depauw, O. Richard, H. Bender, I. Gordon, G. Beaucarne, J. Poortmans, R. Mertens, J.-P. Celis, Study of pore reorganization during annealing of macroporous silicon structures for solar cell application, Thin Solid Films 516 (2008), 6934-6938.

[26] M. Garín, T. Trifonov, A. Rodríguez, R. Alcubilla, Infrared thermal emission in macroporous silicon three-dimensional photonic crystals, Appl. Phys. Lett. 91 (2007), 181901.

[27] M. Garín, T. Trifonov, A. Rodríguez, R. Alcubilla, F. Marquier, C. Arnold, J.-J. Greffet, Improving selective thermal emission of three-dimensional macroporous silicon through porosity tuning, Appl. Phys. Lett. 93 (2008), 081913.

[28] M. Garín, T. Trifonov, D. Hernández, A. Rodríguez, R. Alcubilla, Thermal emission of macroporous silicon chirped photonic crystals, Optics Letters 35 (2010), 3348-3350.

[29] F. E. Nicodemus, Directional Reflectance and Emissivity of an Opaque Surface, Appl. Opt. 4 (1965), 767-773.

[30] J.-J. Greffet, M. Nieto-Vesperinas, Field theory for generalized bidirectional reflectivity: derivation of Helmholtz's reciprocity principle and Kirchhoff's law, J. Opt. Soc. Am. A 15 (1998), $2735-2744$.

[31] H. E. Revercomb, H. Buijs, H. B. Howel, D. D. LaPorte, W. L. Smith, H. A. Sromovsky, 
Radiometric calibration of IR Fourier transform spectrometers: solution to a problem with the HighResolution Interferometer Sounder, Applied Optics 27 (1988), 3210—3218.

[32] C. H. Seager, M. B. Sinclair, J. G. Fleming, Accurate measurements of thermal radiation from a tungsten photonic lattice, Appl. Phys. Lett. 86 (2005), 244105.

[33] J. B. Pendry, L. Martín-Moreno, F. J. Garcia-Vidal, Mimicking Surface Plasmons with Structured Surfaces, Science 305 (2004), 847-848.

[34] A. Datas, C. Algora, Detailed balance analysis of solar thermophotovoltaic systems made up of single junction photovoltaic cells and broadband thermal emitters, Solar energy materials \& solar cells 94 (2010), 2137-2147. 


\section{Figure captions}

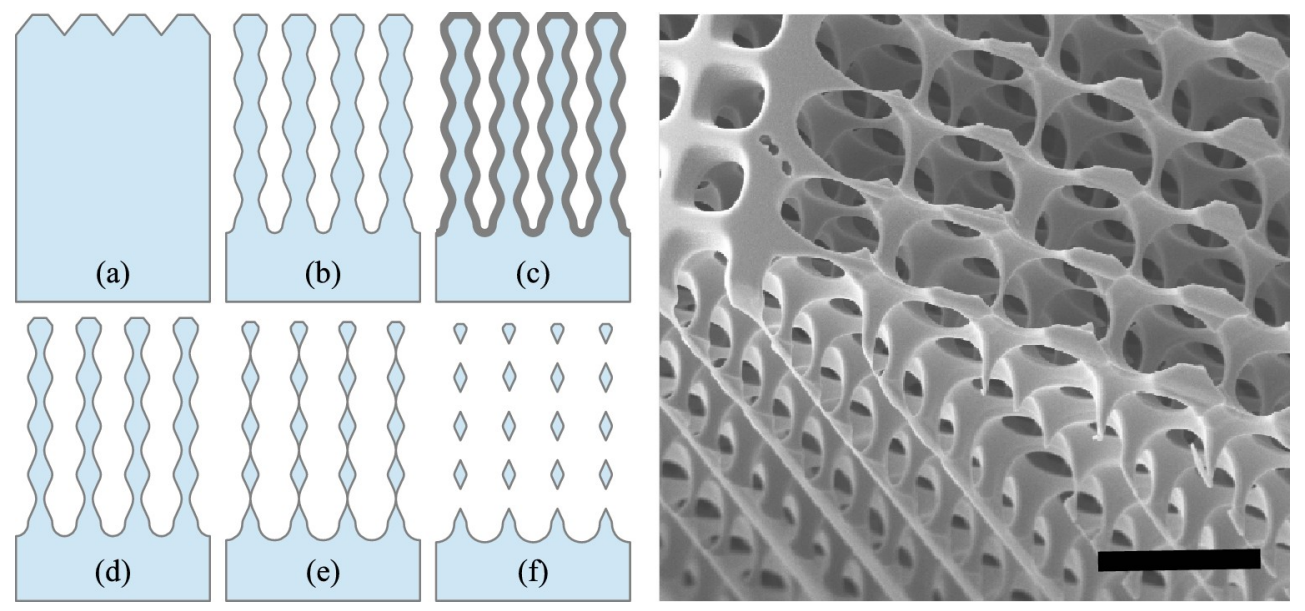

Fig 1. Schematic of the scaffold fabrication. (a) Pore nucleation. (b) Electrochemical etching. (c,d) oxidation/oxide-strip widening cycle. $(\mathrm{d}-\mathrm{f})$ Increased porosity after several cycles. The SEM image shows a highly-porous final structure. Scale bar is $4 \mu \mathrm{m}$.

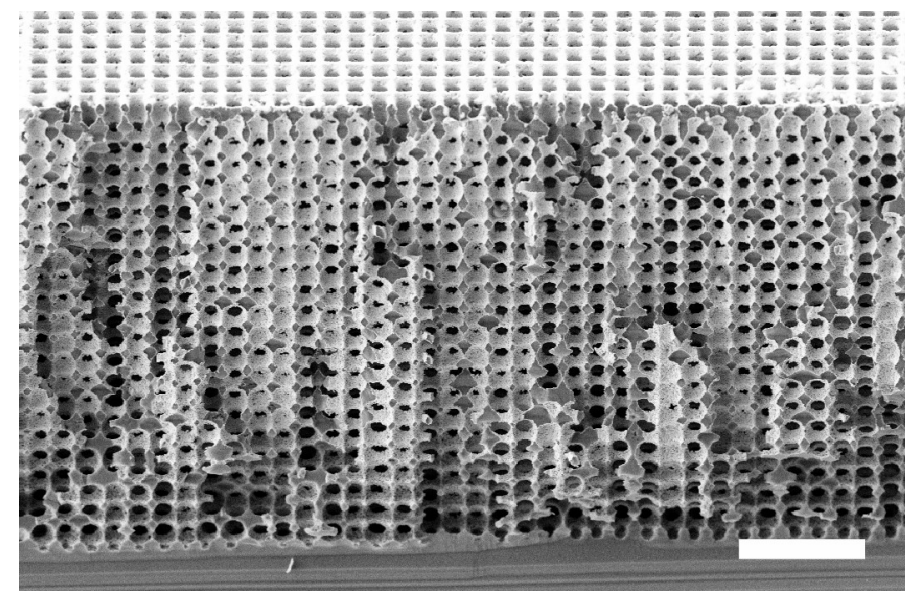

Fig. 2. SEM image, broken side, of a final $\mathrm{Si} / \mathrm{Pt}$ selective emitter. The ALD Pt film covers the full 3D structure. Scale bar is $10 \mu \mathrm{m}$. 


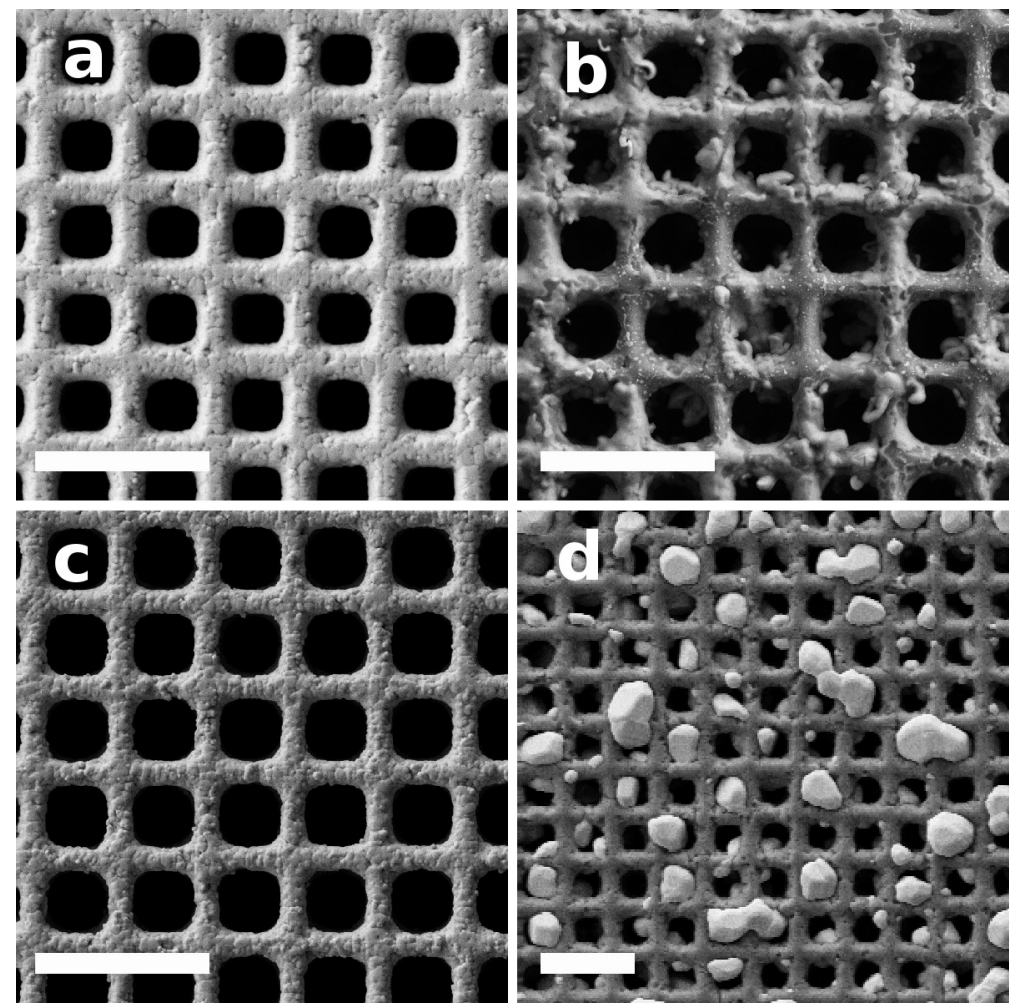

Fig. 3. Effect of temperature on the sample's morphology. (a) Top surface of a $\mathrm{Si} / \mathrm{Pt}$ sample before annealing. (b) Surface of a Si/Pt sample after annealing $8 \mathrm{~h}$ at $1100 \mathrm{~K}$ in pure $\mathrm{N}_{2}$ ambient. (c) $\mathrm{A} \mathrm{Si} / \mathrm{SiO}_{2} / \mathrm{Pt}$ sample after annealing $8 \mathrm{~h}$ at $1100 \mathrm{~K}$ in pure $\mathrm{N}_{2}$ ambient. (d) $\mathrm{A}$ $\mathrm{Si} / \mathrm{SiO} 2 / \mathrm{Pt}$ sample after $12 \mathrm{~h}$ annealing at $1273 \mathrm{~K}$ in ambient air. Scale bar is $4 \mu \mathrm{m}$. Panel (c) represent our best stability results. 


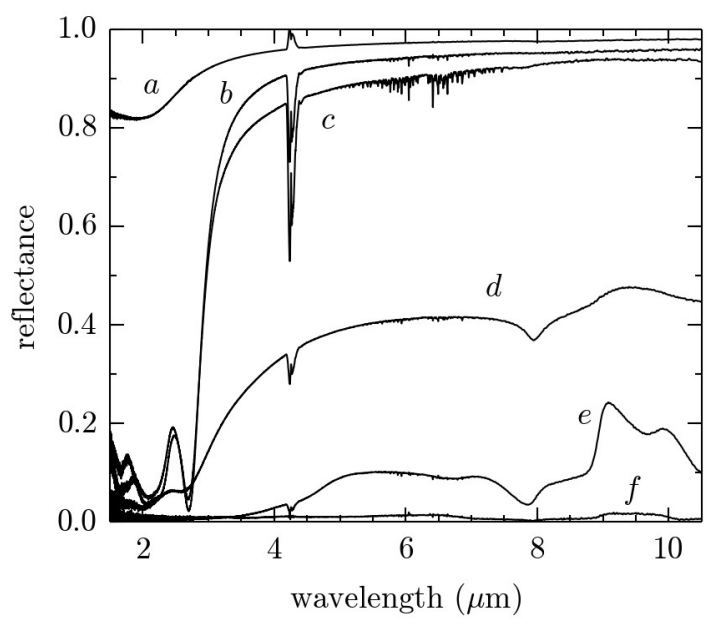

Fig. 4. Quasi-normal $\left(13^{\circ}\right)$ reflectance for different structures after different annealing tests. (a) Reflectance of 50nm Pt over polished Si for reference purposes. (b) Si/Pt sample before and after $8 \mathrm{~h}$ annealing at $1000 \mathrm{~K}$ in $\mathrm{N}_{2}$ ambient (they are indistinguishable). (c) $\mathrm{Si} / \mathrm{SiO} 2 / \mathrm{Pt}$ sample after $8 \mathrm{~h}$ at $1100 \mathrm{~K}$ in $\mathrm{N} 2$. (d) $\mathrm{Si} / \mathrm{Pt}$ sample after $8 \mathrm{~h}$ at $1100 \mathrm{~K}$ in $\mathrm{N}_{2}$. (e) $\mathrm{Si} / \mathrm{SiO}_{2} / \mathrm{Pt}$ after $12 \mathrm{~h}$ at $1273 \mathrm{~K}$ in ambient air. (f) $\mathrm{Si} / \mathrm{Pt}$ sample after $12 \mathrm{~h}$ at $1273 \mathrm{~K}$ in ambient air. Notice that curve (c) represent our best stability results.

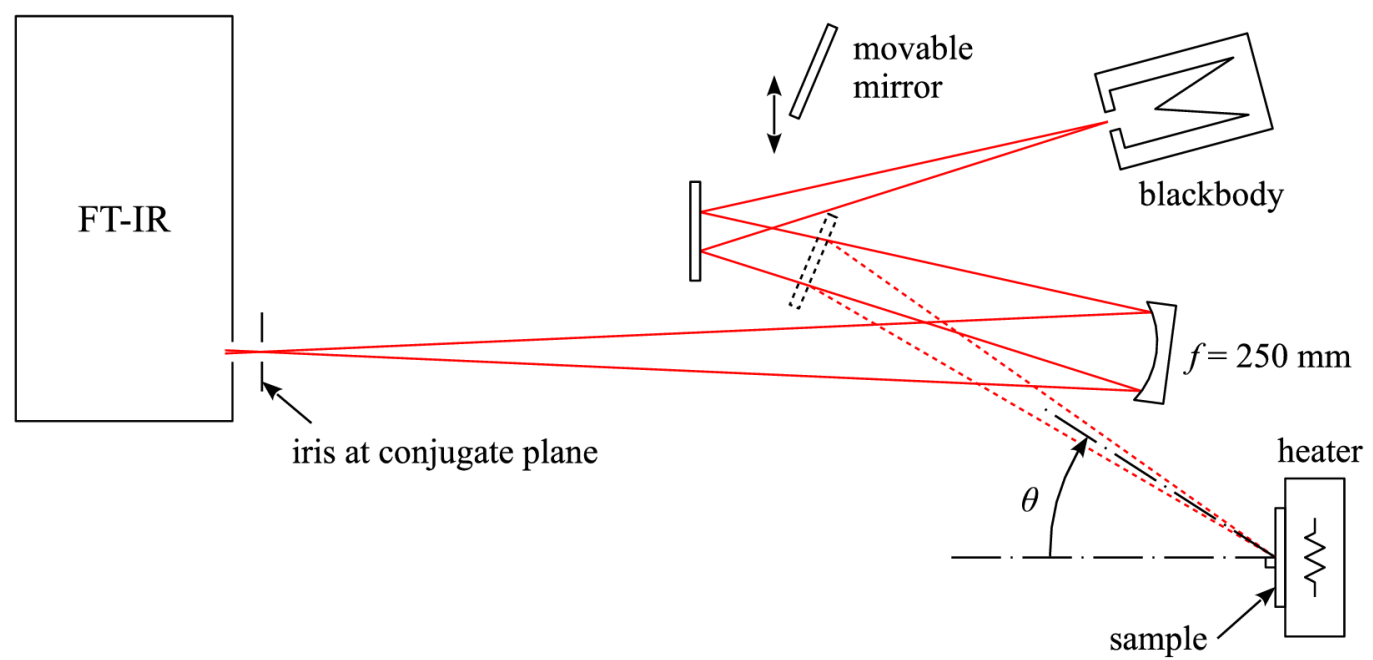

Fig. 5. Schematic of the set-up for measuring the thermal emission spectrum at high temperature. 

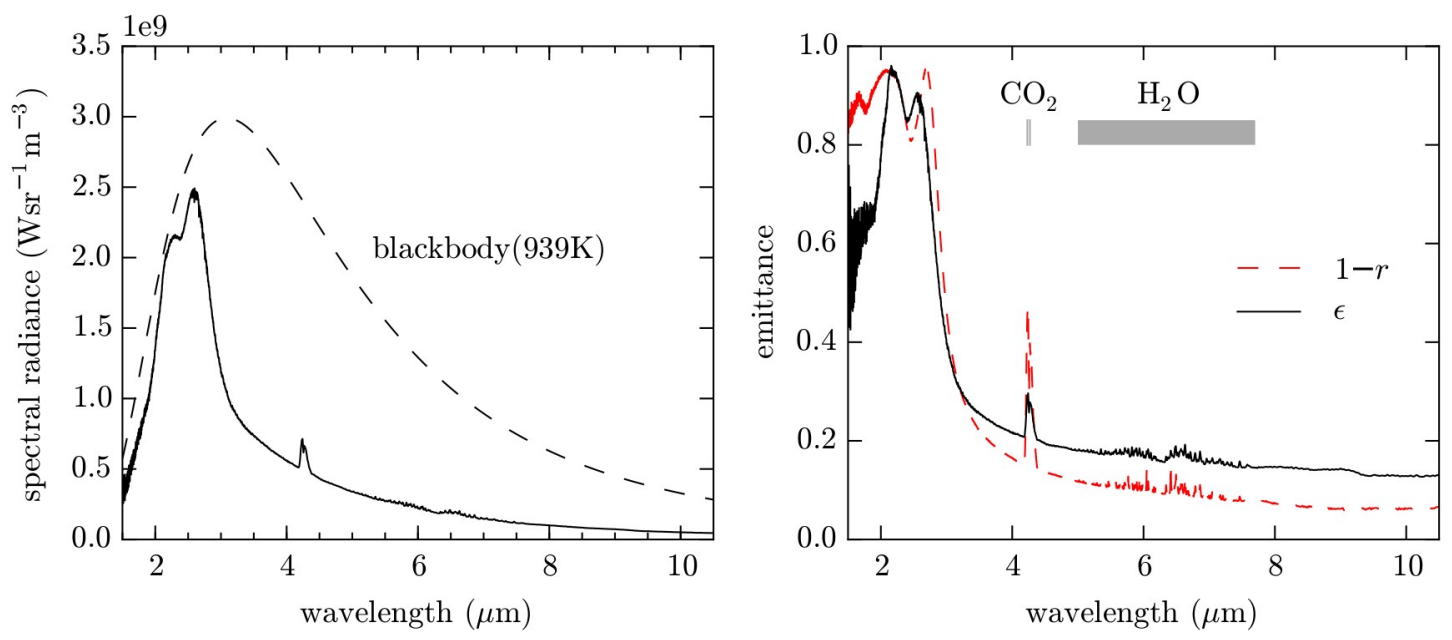

Fig. 6. Normal spectral radiance (left) and normal spectral emissivity (right) of a $\mathrm{Si} / \mathrm{SiO}_{2} / \mathrm{Pt}$ structure measured at $939 \mathrm{~K}$. The $1-r$ curve (dashes), measured at room temperature and at quasi-normal incidence angle, is also shown in the right figure for comparison purposes. This sample was previously annealed $8 \mathrm{~h}$ at $1100 \mathrm{~K}$ in pure $\mathrm{N}_{2}$ ambient.
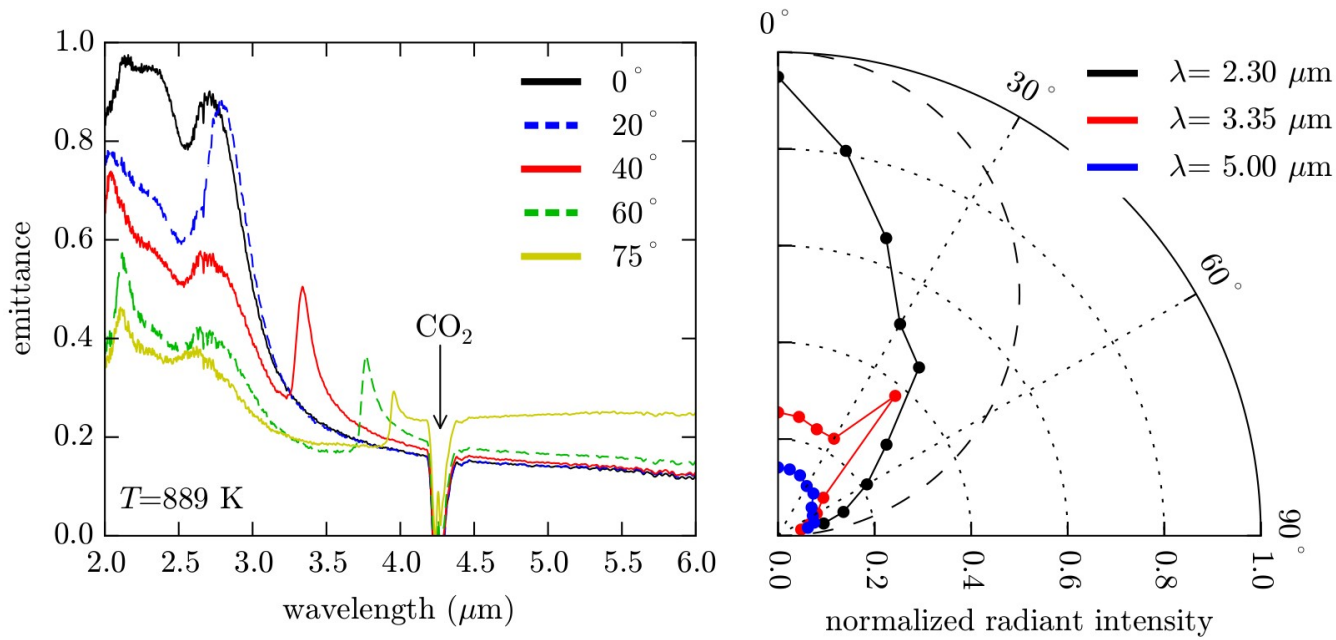

Fig. 7. (left) Spectral emittance measured at $890 \mathrm{~K}$ for different exit angles. (right) Normalized radiant intensity polar plot for several wavelengths. The dashed line represents the black-body curve. 

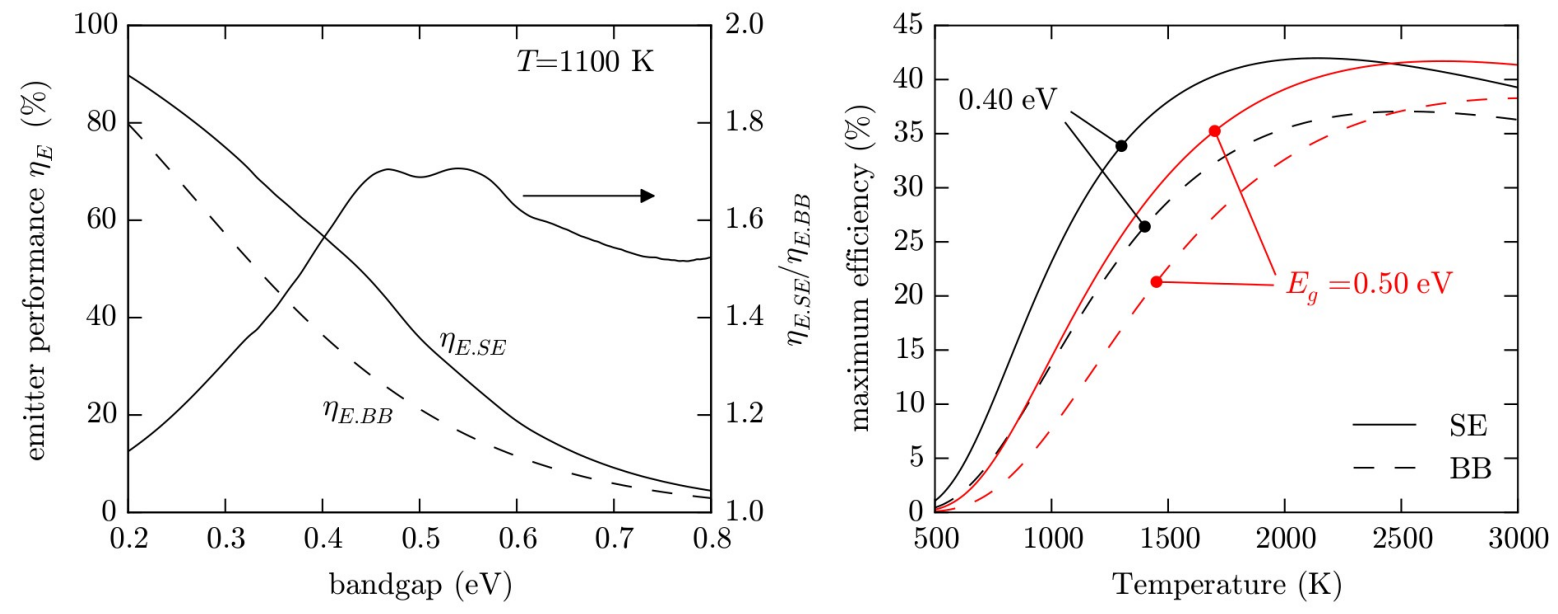

Fig. 8. (left) Emitter performance, $\eta_{E}$, as a function of the PV cell bandgap for the fabricated selective emitter, $\eta_{\mathrm{E} . \mathrm{SE}}$, at $T=1100 \mathrm{~K}$. The performance of a blackbody emitter, $\eta_{\mathrm{E} . \mathrm{BB}}$, is also shown for comparison in dashed line. (right) Maximum theoretical TPV system efficiency as a function of the emitter temperature for two different PV cell band gap. The efficiencies achievable with a BB emitter, dashed lines, is also shown for comparison. All curves have been calculated considering the experimental emittance spectra shown in fig. 6 and 7. 